International Journal of Biomedicine | June 2019 - Volume 9, Issue Suppl_1: Abstracts From the Second Russian International Conference "Cryo-electron microscopy 2019: achievements and prospects"

ORAL ABSTRACT PRESENTATIONS

SESSION TITLE: STRUCTURE OF VIRUSES AND CHAPERONINS

DOI: 10.21103/IJBM.9.Suppl_1.OR9

\title{
Abstract OR-9: Cryo-Electron Microscopy Studies of Flaviviruses
}

Victor A. Kostyuchenko, Guntur Fibriansah, Shuijun Zhang, Thiam-Seng Ng, Shee-Mei Lok

Virus structure and function group, Program in Emerging Infectious Diseases, Duke-NUS Medical School, Singapore

Background: Flaviviruses cause human disease such as dengue fever, West Nile fever, and Zika fever with high degree of severity. There are almost no licensed vaccines and treatment except symptomatic for these diseases. Our group studies the virus structure and interactions with ligands such as receptors and antibodies in order to understand the virus' life cycle with the aim to find possible preventive or curative drugs.

Methods: For structural biology work, we employ mostly cryo-EM such as single particle analysis and cryo-electron tomography to study complexes of the virus with antibody fragments.

Results: We obtained subnanometer and near-atomic resolution cryo-EM structures for immature and mature forms of dengue virus serotype 1, dengue virus serotype 4, and zika virus. We described a possible maturation pathway based on the higher resolution structures. We also described a temperaturedependent structural change that occurs in some strains of the flaviviruses, important for vaccine development. In addition, we described a cryo-EM structure of a complex of a dengue virus and antibody fragment that demonstrates that in some cases cryo-EM is superior to X-ray crystallography as providing a fuller picture of the virus and antibody interaction.

Conclusion: Modern cryo-EM allows us a better and more detailed look at the viruses, their life cycle and help the development of a better vaccines and therapeutics.

Key Words: Dengue virus • Zika virus • Flavivirus • Cryo-EM

Sources of Funding: This work was supported by grants from Singapore Ministry of Education and National Medical Research Council to Prof. Lok.

International Journal of Biomedicine. 2019;9 Suppl 1: S9. doi: 10.21103/IJBM.9.SuppI_1.OR9

C2019 International Medical Research and Development Corporation 